\title{
Diagnostic tools in male infertility—-the question of sperm dysfunction
}

\author{
Christopher LR Barratt ${ }^{1,2}$, Steven Mansell ${ }^{1}$, Catherine Beaton ${ }^{1}$, Steve Tardif $^{1}$ and Senga K Oxenham ${ }^{1}$
}

Sperm dysfunction is the single most common cause of infertility, yet what is remarkable is that, there is no drug a man can take or add to his spermatozoa in vitro to improve fertility. One reason for the lack of progress in this area is that our understanding of the cellular and molecular workings of the mature spermatazoon is limited. However, over the last few years there has been considerable progress in our knowledge base and in addressing new methods to diagnose sperm dysfunction. We review the current state of the field and provide insights for further development. We conclude that: (i) there is little to be gained from more studies identifying/categorizing various populations of men using a basic semen assessment, where an effort is required in making sure the analysis is performed in an appropriate high quality way; (ii) technological development is likely to bring the reality of sperm function testing closer to implementation into the clinical pathways. In doing this, these assays must be robust, cheap (or more appropriately termed cost effective), easy to use and clinically useful; and (iii) clinical necessity, e.g., the need to identify the highest quality spermatozoon for injection is driving basic research forward. This is an exciting time to be an andrologist and, likely, a fruitful one.

Asian Journal of Andrology (2011) 13,53-58; doi:10.1038/aja.2010.63; published online 22 November 2010

Keywords: gamete biomarker; male fertility; sperm biomarker; sperm dysfunction

\section{INTRODUCTION}

Infertility is a significant global problem affecting approximately 80 million (1:7) couples worldwide. ${ }^{1}$ In a landmark study by Mike Hull and colleagues, in which a representative British population was studied, sperm dysfunction (lacking 'normal' function) was identified as the single most common cause of infertility. ${ }^{2}$ Subsequent studies have confirmed these observations $s^{3}$ and highlighted dysfunctional cells in men with 'normal' semen parameters and conversely normal sperm function in oligozoospermic men. ${ }^{4}$ What is remarkable is that, for this group, there is no drug a man can take or add to his spermatozoa in vitro to improve fertility. The only option is assisted reproductive technology (ART) which usually consists of a graduation of treatment depending on severity, i.e., intrauterine insemination for mild, in vitro fertilisation (IVF) for moderate and intracytoplasmic sperm injection (ICSI) for men with severe sperm dysfunction. One reason for the lack of progress in this area is that our understanding of the cellular and molecular workings of the mature spermatazoon is limited. However, over the last few years there has been considerable progress in our knowledge base and in addressing new methods to diagnose sperm dysfunction. The purpose of this paper is to review the current state of the field and provide insights for further development. The initial focus is on the value of semen analysis as a clinical tool with the discussion progressing to examining sperm dysfunction in detail.

\section{WHERE IS SEMEN ANALYSIS NOW AND WHERE IS IT GOING?}

The recent fifth edition of the WHO semen analysis manual ${ }^{5}$ addresses many of the criticisms levelled at previous versions ${ }^{6}$ and now includes step by step methods, constructive discussion of quality control and quality assurance, detailed descriptions of the assessment of sperm morphology and, for the very first time, biologically based references ranges. The WHO manual has always been the bedrock of andrology and the fifth version is likely to assist the development of the field still further. However, based on previous experiences, major challenges remain to be addressed. ${ }^{7}$ These have continually been present yet, the evidence to date suggests that although the problems can be easily identified there is often minimal or no resolution. Three particular aspects merit further attention.

Firstly, technicians are not using the detailed laboratory methods even when provided in comprehensive manuals. ${ }^{8}$ An example of this is the review of practice in the UK. ${ }^{9}$ Remarkably, in a country that has had semen training course for many years, only 5\% of laboratories carried out the WHO standard method for morphology assessments. Equally surprising was that $69 \%$ of laboratories counted $\leqslant 100$ sperm for morphology assessments making the assay effectively redundant. This poor practice is of course not limited to the UK and has been highlighted by a number of authors. ${ }^{10,11}$

Secondly, training methods exist—but are they used/useful? A plethora of data shows that there are robust training methods available for assessment of basic semen analysis. ${ }^{12}$ A series of programs have been developed and proven to reduce variability. ${ }^{13}$ What these programs show is: (i) technicians currently performing semen assessments often produce great variability; (ii) proven techniques and intensive instruction can improve education and reduce variability dramatically; and (iii) with ongoing training, even when the technicians return back to

${ }^{1}$ Reproductive and Developmental Biology, Maternal and Child Health Science Laboratories, Centre for Oncology and Molecular Medicine, Ninewells Hospital, University of Dundee, Dundee, DD1 9SY, Scotland, UK and ${ }^{2}$ Assisted Conception Unit, NHS Tayside, Ninewells Hospital, Dundee, DD1 9SY, Scotland, UK Correspondence: Dr C L R Barratt (c.barratt@dundee.ac.uk)

Received: 18 October 2010; Revised: 24 October 2010; Accepted: 25 October 2010; Published online: 22 November 2010 
their home laboratory environment, standards can be maintained. However, what is observed from, for example, external quality control programs is that the majority of laboratories have a great deal of error in their analysis. ${ }^{14}$ Clearly there is a discourse here-training works, but the clinical assessments are often poor. The reasons behind this discourse are unclear. One possibility is that although methods to train technicians exist, there are not enough courses available for the vast number of people doing semen assessments and/or technicians are not attending these courses. The alternative hypothesis is that traditional teaching methods are not the way to address the problem. It is very likely that demand for intensive courses is not being met for a variety of economical and practical reasons and clearly this needs to be urgently addressed. However, newer methods and/or structures of training laboratory andrologists, e.g., via the Internet must be developed. Complementary training can be instigated by using a sophisticated laboratory book system which allows comprehensive detailed supervised training on site. An example is the structure adopted by the Association of Biomedical Andrologists (www.aba.uk.net). The hypothesis (and hope) is that the improvements in the training of staff will be translated into higher standards of semen assessments.

Thirdly, consistently both scientists and clinicians in our discipline denigrate the value of semen assessments with arguments based on a series of false assumptions. ${ }^{11,14,15}$ An example is the assumption that semen assessments are currently performed in an adequate manner. This is wrong. The evidence from external quality control schemes demonstrates that some patients will be referred for inappropriate treatment, e.g., ICSI when they may not even need ART. In simplistic terms, we are exposing a large number of couples to inappropriate financial and psychological stress. Additionally, we are potentially exposing the female to harmful procedures, e.g., IVF. Perhaps this third lesson will not be executed until legal action ensues.

\section{WHY DOES MALE INFERTILITY REQUIRE MORE ROBUST DIAGNOSTIC TOOLS THAN A BASIC SEMEN ANALYSIS?}

Although the diagnostic and predictive value of traditional semen parameters has been debated for over 80 years, the inescapable conclusion remains that its clinical value is limited. The remits of this limitation are contentious but two facts are clear: (i) at the lower ends of the spectrum, e.g., low concentrations of motile spermatozoa, there are significantly higher chances of subfertility; ${ }^{16,17}$ and (ii) except in rare cases, e.g., globozoospermia, ${ }^{18}$ values above these limits provide minimal diagnostic clarity. ${ }^{19,20}$ This information is not new and was highlighted by MacLeod and colleagues who concluded that 'The greatest difference between the two groups (infertile and fertile) is seen at the count levels under 20 million/cc. Only $5 \%$ of fertile men compared with $16 \%$ of the 'infertile' group fall into this category'. ${ }^{21}$ Importantly, for the robustness of the conclusions, a plethora of subsequent studies have repeated these experiments in various guises and, not surprisingly, come to the similar conclusions. The most recent of these, undertaken by the WHO, ${ }^{22}$ provide new reference values for semen assessment which not surprisingly are remarkably similar (with exception of morphology) to that proposed by MacLeod and Gould in $1951 .{ }^{21}$

Therefore, the conclusions regarding the clinical relevance of semen assessment are unlikely to change. New studies are not required. What are urgently needed are new assessments of male reproductive potential. This is the focus of the following discussion.

\section{SPERM DYSFUNCTION-OLD TESTS LYING DORMANT?}

To date, the assessment of sperm function has failed to make a significant impact on the clinical management of couples. The reasons for this, e.g., lack of standardized protocols have been rehearsed elsewhere. ${ }^{20}$ In summary, the logarithmic progress that has been made in understanding the basic science around how a spermatozoon develops, prepares for fertilisation and contributes to a healthy birth has not (yet) been translated into routine clinical practice. ${ }^{23}$

It is disappointing to arrive at this conclusion, but it is heartening to realize that this is very likely to change in the near future. One example will suffice. A primary functional assay is the human zona-binding test. Whilst the paradigm of sperm binding and subsequent acrosome reaction may be questioned in mice, ${ }^{24}$ consistently, robust clinical data demonstrates its usefulness for ART success. A large range of patients have been identified with poor or adequate binding but minimal induction of the acrosome reaction in response to the zona. ${ }^{25}$ However, a key problem with the widespread use of human zonabinding assays, in fact its universal restriction, is the availability of material. The initial promise of using recombinant zona pellucida protein 3 (ZP3) as a surrogate for the human zona was not fulfilled primarily because: (i) techniques to produce the recombinant proteins were not optimized; and (ii) our knowledge base of what proteins were present in the zona and their structures was unavailable. These points have and are being addressed (respectively). Exciting data from a series of experiments using what appears to be robust preparations of recombinant human zona proteins has been published. ${ }^{26}$ An in vitro sperm function assay using recombinant products is now much more of a reality.

Whilst the objective of robust assays of sperm function must inevitably be to simplify the tools for routine use, we are dealing with complex systems; thus, the challenges in developing these assays are considerable. An example of this is the regulation of calcium by the cell which is clearly critical for key physiological processes, e.g., motility and acrosome reaction. Whilst our knowledge of how calcium is regulated in the cell has increased substantially, ${ }^{27,28}$ there are still fundamental gaps, e.g., the role of the putative calcium stores in the sperm neck/midpiece region. However, decades of clinical research suggest that calcium regulation can potentially be used as a tool for identifying dysfunctional cells. ${ }^{29}$ What are missing are robust assays. In our laboratory, we have been using an FLUOstar assay (BMG labtech. Offenburg, Germany) for the rapid screening of calcium mobilisation in prepared semen samples and although the assays are accurate and rapid, there are practical problems with their routine use. For example, currently it requires a minimum of 0.5 million cells post preparation to obtain robust data points. This excludes a number of men referred for ART where we have no information and arguably (see above); these are the men who have the most to gain from detailed sperm function testing. Additionally, currently progesterone is used as an agonist to induce a response and although there is considerable clinical data to support its use, ${ }^{30}$ we have minimal knowledge of what progesterone actually does in the cell, e.g., mobilisation of stores, interacting with the receptors. Maybe other agonists would provide better tools, but they have yet to be indentified. Abnormalities in signalling do exist, but presently we do not have a reliable method to assess these problems and although progress in basic research is very impressive, we need to complement this with a translational focus.

\section{SPERM FUNCTION TESTING AND ICSI}

There has been a plethora of studies suggesting that, in the overwhelming majority of cases, the quality of the semen has little or no influence on the success rates of ICSI. ${ }^{31}$ Not surprisingly, there is a lack of data on sperm function testing and the focus of activity has been in attempting to improve the selection process of spermatozoa, e.g., 
hyaluronic acid binding (Table 1). However, it is increasingly apparent that studies are hampered by the lack of knowledge of what defines a functional spermatozoon and the destructive nature of the investigations. This results in extensive data on surrogate quality indicators of entire populations but fails to address the individual quality of a single spermatozoon. As a result, a wide variety of techniques have been tried based on current knowledge in the hope of improving pregnancy outcomes. These include externalisation of phosphotidylserine (magnetic-activated cell sorting), ${ }^{32}$ cell charge (zeta charge), ${ }^{33}$ maturity markers (hyaluronic acid binding) ${ }^{34-36}$ and detailed morphological analysis (intracytoplasmic morphologically selection sperm injection)..$^{37-40}$. Although some studies have recorded significant improvements in clinical pregnancy rates, these studies generally fail to present useful statistical data that will indicate the effectiveness of this method over conventional ICSI. Such data include, number needed to treat (number of oocytes injected before pregnancy is achieved compared to the control), relative risk (the risk of falling pregnant using this method compared to the control) and odds ratio (the odds of falling pregnant compared to the control). As well as this, the lack of studies reporting live birth rates is noticeable, preventing the assessment of the take home baby rate and the overall effectiveness of the method. Another question is what levels of improvement is truly significant, $2 \%$ or $20 \%$ and are these new methods applicable to a busy IVF laboratory both financially and logistically.

Another way to look at this problem is to address if, and how, sperm are capable of confessing their errors/flaws externally, ${ }^{41}$ which may allow non-invasive sperm selection, e.g., in response to DNA damage

${ }^{42}$. However, it remains to be seen whether such biomarkers do exist or if the heterogeneity of spermatozoa is too great for such selection methods to be applicable to every patient. Instead, case-by-case assessments may have to be used, adding time and additional effort. What is certain is that a clear clinical requirement (identification of the 'best' spermatozoon) is acting as a welcome catalyst and focus on the detection of high quality cells in ART will continue to have a direct effect on the proliferation of basic research in the area.

\section{THE USEFULNESS OF ANIMAL STUDIES: ZONADHESIN AS AN EXAMPLE}

With relatively few exceptions the data from mouse knockout studies has not been successfully translated into the clinical arena. There are a number of reasons for this with differences in the reproduction process being one; ${ }^{43}$ however, with respect to sperm dysfunction, there are sufficient key similarities between mice and men to provide a more in depth understanding and the potential to identify candidate proteins to be used as a biomarker of dysfunction. An example is the intraacrosomal protein zonadhesin.

Zonadhesin was first discovered in pigs based on the ability to bind the ZP and subsequent studies identified it in mouse, hamster, horse, donkey, zebra, rat, guinea pig, chinchilla, dog and human (Tardif et al., unpubl. data). ${ }^{44-47}$ Importantly, sperm-ZP adhesion activity confers species specificity and to date, zonadhesin is the only sperm protein among sperm-ZP adhesion candidates showing species specificity during fertilisation. This was established by the high affinity of zonadhesin for native ZP between homologous gametes and, more recently, using zonadhesin-null mice. ${ }^{48}$ Interestingly, mouse zonadhesin was only detectable at the sperm surface of live spermatozoa after incubation in conditions supporting sperm capacitation. Preliminary data suggest that the same is true for human cells and the question is - can this intra-acrosomal protein be used as a potential biomarker for human sperm function/dysfunction?

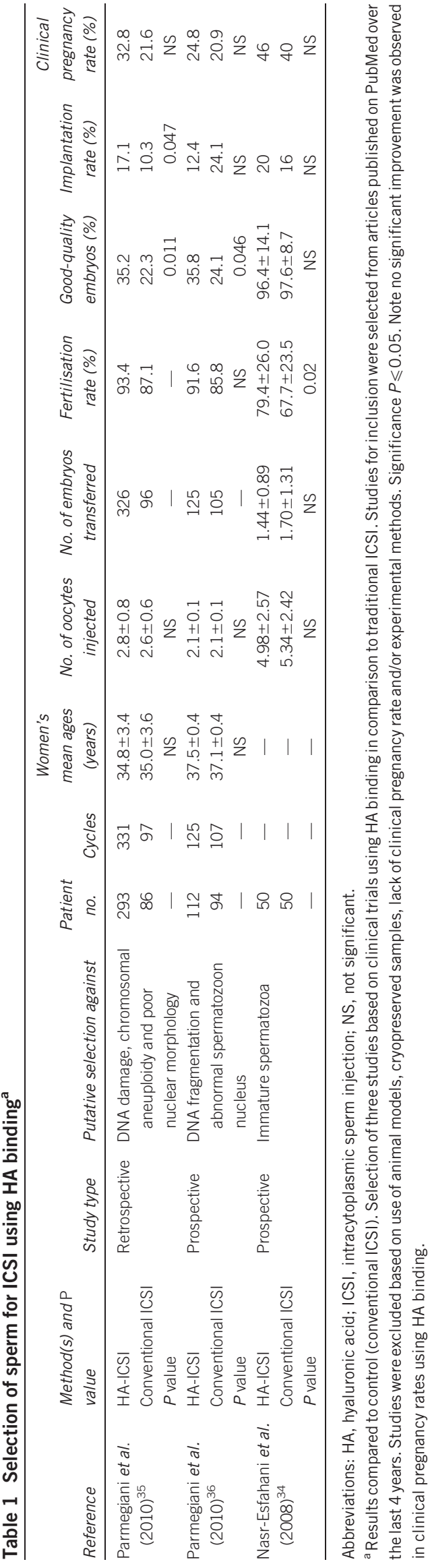


Based on the zonadhesin exposure during capacitation in the mouse, we proposed a putative model (Figure 1), where human zonadhesin could be exposed after capacitation (or latter stages of) differently between fertile and subfertile men. Zonadhesin exposure could be reduced in spermatozoa unable to undergo capacitation due possibly to problems in protein trafficking. Conversely, under noncapacitating conditions zonadhesin exposure would not be expected or only present in a very small percentage of cells, but in some subfertile men there may be premature exposure due to a misregulation of transduction events.

Currently there is a paucity of markers of human sperm function at a molecular level. One variation between fertile and subfertile men could reside in how the proteins are post-translationally modified as opposed to their mere presence or absence. In this context we have observed, in two different subfertile patients who experienced failed fertilisation at IVF, significantly different polypeptide distribution and processing of zonadhesin compared to controls (Tardif et al., unpubl.

a

Non-capacitating condition

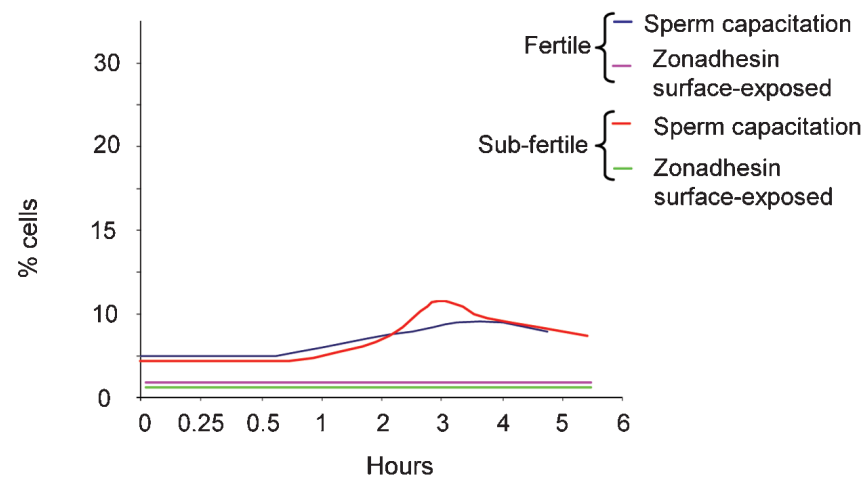

b Capacitating condition

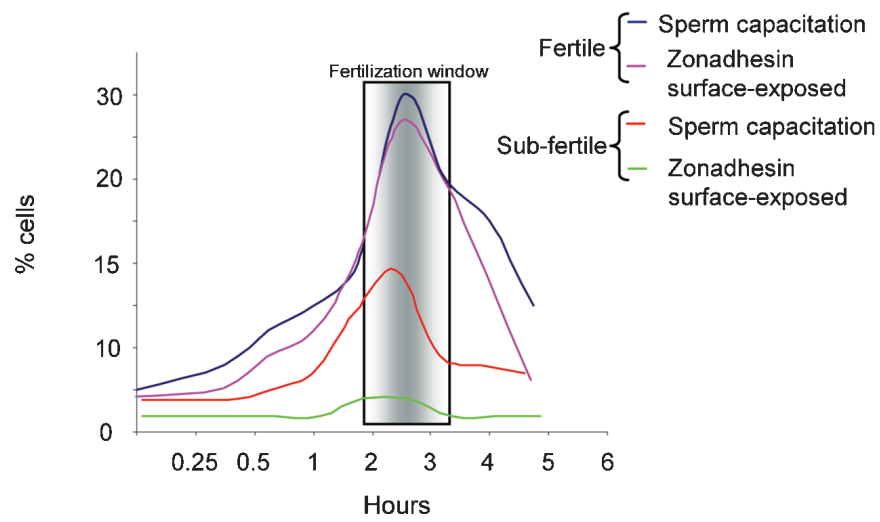

Figure 1 Putative model of human zonadhesin exposure during sperm incubation under (a) non-capacitating or (b) capacitating conditions in fertile and subfertile men. Diagram indicates time course of sperm capacitation (red and blue) and zonadhesin exposure (pink and green) in fertile and subfertile men respectively. This model suggests that sperm capacitation is closely related to zonadhesin exposure in fertile men; however, a decrease of zonadhesin exposure could be associated with subfertile men related to compromised capacitation. Shaded area in (b) represents the fertilisation window. data). Whilst preliminary, our data suggest that zonadhesin has the potential to be a biomarker for evaluating a fertile sperm population.

\section{POTENTIAL NEW PARADIGMS-HOME TESTING FOR MALE INFERTILITY}

An area which has seen considerable progress is the development of a number of putative home sperm tests. ${ }^{49-51}$ The details of these and arguments for and against their use are presented elsewhere, ${ }^{20,51}$ but for widespread acceptance these assays need to be: (i) testing some functional capacity of the cell rather than numbers; (ii) robust; (iii) cheap; and (iv) widely available. Whilst currently no test fulfils all of these criteria, with the rapid developments in new technologies, it is likely that new and more robust versions of home test will become available in the very near future. Interestingly, these tests may also be taken a step further-from diagnosis to treatment. For example, they may indicate that the man has sperm present but that these cells are functionally defective, e.g., produce high levels of lipid peroxidation end products. If antioxidant therapy is, in the future, a proven treatment for oxidative stress, then it is feasible that a man could have a diagnosis and order (self-) treatment (for example vitamin E) all within one hour! Although exciting, this is at present a fantasy. Critically, what has to be established is where in the patient's pathway these assays will be of most benefit? The original hypothesis was that they would be the first stage for a couple who were enquiring about their fertility. ${ }^{20,51}$ Following the test, the couple could make a choice (depending on the result) to continue trying or seek earlier referral. The logic was that seeking earlier referral would increase the chances of conception particularly in couples where female age was a pressing problem. However, whilst attractive, this concept remains untested and although patients may find home testing very appealing, there is as yet no direct evidence that over the counter tests would improve the chances of conception.

\section{FUTURE MARKERS OF SPERM DYSFUNCTION-NEW TECHNOLOGIES}

This is a particular exciting time in andrology. For example, following the somewhat controversial discovery of mRNA in mature human sperm, ${ }^{52}$ there have been a number of studies suggesting that these mRNAs could be used as a diagnostic tool-equivalent to a transcriptome. ${ }^{53}$ Whilst the data to support this is in its infancy, this is likely to be an area of rapid development which holds great promise.

The power of proteomics is now increasingly being applied to mature spermatozoa. ${ }^{54}$ Although technical difficulties are preventing the complete sperm proteome from being available, ${ }^{55}$ the cataloguing to date is impressive and consistently brings new thoughts to the field on how the cell works. ${ }^{56}$ Comparison to the proteome of other species could answer fundamental questions such as: what is the basic machinery necessary to make a functionally mature male gamete ${ }^{57}$ Complementary to completing the tool kit of the sperm proteome, another area where proteomics is having an impact is the identification of putative candidate biomarkers of high- and poor-quality cells. Impressive data are rapidly appearing, for example comparing potential differences in men with specific pathologies, e.g., asthenozoospermia. ${ }^{58}$

Driven by the overwhelming clinical need to identify subfertile men without the requirement for a semen assessment, we may soon be able to obtain metabolomic profiling on blood samples which act as a surrogate for fertility. This is a very difficult area as comparable experiments in other disciplines, e.g., cancer highlights the herculean challenges in the identification of robust candidates of dysfunction which can be readily translated into clinical practice. ${ }^{59}$ However, this is 
potentially very exciting. Additionally, other techniques such as Raman spectroscopy are beginning to be used to identify (noninvasively) genetically compromised cells driven by the imperative to inject the most genetically functional cell at ICSI. ${ }^{60}$

Whatever new tests of sperm function, or reworking of old ones are proposed, we need to heed the lessons of the past. A classic example is the sperm chromatin/DNA damage assays. The primary data supporting these was presented in 1980 and although they are consistently suggested as important tools in the armamentarium, they face considerable challenges before they can become part of routine clinical management. ${ }^{61}$

\section{SUMMARY - WHERE TO GO NOW?}

The arguments presented suggest that for semen analysis, there is little to be gained from more studies identifying/categorizing various populations of men. Where an effort is required is making sure the analysis is performed in an appropriate high-quality way.

Technological development is likely to bring the reality of sperm function testing closer to implementation into the clinical pathways. In doing this, these assays must be: (i) robust; (ii) cheap (or more appropriately termed cost effective); (iii) easy to use; and (iv) clinically useful. To achieve the last tenet is a major challenge that requires special consideration. If these assays are to be routinely used, there must be a rigorous examination of their application using standardized procedures. ${ }^{62}$ Additionally, there needs to be a critical determination of where these assays fit within the patient pathway. ${ }^{63}$ To date, assays of the functional competence of sperm have not yet been evaluated in this critical manner. Real progress depends on enforcing these tenets.

There is a palpable excitement in andrology. Importantly, clinical requirements are acting as a catalysts and focus on the detection of high-quality cells in ART which is having a direct effect on the proliferation of basic research. Additionally, there are very clear advances in our understanding of the mature cell in no small part being brought about by application of proven technology from other areas. This is an exciting time to be an andrologist and, likely, a fruitful one.

\section{AUTHOR CONTRIBUTIONS}

CLRB designed the concept and framework of the paper and wrote the first draft. All authors contributed to the drafting and final editing of the manuscript.

\section{COMPETING FINANCIAL INTERESTS}

The authors declare no relevant commercial interest.

\section{ACKNOWLEDGMENTS}

Work in the author's laboratories is funded by The Wellcome Trust, TENOVUS (Scotland), University of Dundee, MRC (Developmental Pathway Funding Scheme), NHS Tayside and Scottish Enterprise.

The authors are very grateful to all members of the Assisted Conception Unit at Ninewells Hospital for assistance with obtaining donors and patient's samples for research and training purposes. The senior author (CLRB) is very grateful to W. Christopher L. Ford for many interesting discussions regarding the concept of sperm function testing.

1 Boivin J, Bunting L, Collins JA, Nygren KG. International estimates of infertility prevalence and treatment-seeking: potential need and demand for infertility medical care. Hum Reprod 2007; 22: 1506-12.

2 Hull MG, Glazener CM, Kelly NJ, Conway DI, Foster PA et al. Population study of causes, treatment, and outcome of infertility. Br Med J (Clin Res Ed) 1985; 291 : 1693-7
3 Irvine DS. Epidemiology and aetiology of male infertility. Hum Reprod 1998; 13 Suppl 1: 33-44.

4 Aitken RJ. Sperm function tests and fertility. Int J Androl 2006; 29: 69-75.

5 World Health Organization. WHO Laboratory Manual for the Examination and Processing of Human Semen. 5th edn. Geneva: WHO Health Organization, 2010. http://www.who.int/reproductivehealth/publications/infertility/9789241547789/en/.

6 Barratt CL. On the accuracy and clinical value of semen laboratory tests. Hum Reprod 1995; 10: 250-2.

7 de Jonge CJ, Barratt CL. WHO manual...who should care? Hum Reprod 1999; 14 2431-3.

8 Mortimer D. Practical Laboratory Andrology Oxford: Oxford University Press: 1994

9 Riddell D, Pacey A, Whittington K. Lack of compliance by UK andrology laboratories with World Health Organization recommendations for sperm morphology assessment. Hum Reprod 2005; 20: 3441-5.

10 Ombelet W, Pollet $\mathrm{H}$, Bosmans $\mathrm{E}$, Vereecken A. Results of a questionnaire on sperm morphology assessment. Hum Reprod 1997; 12: 1015-20.

11 Pacey AA. Quality assurance and quality control in the laboratory andrology. Asian J Androl 2010; 12: 21-5.

12 Björndahl L, Barratt CL, Fraser LR, Kvist U, Mortimer D. ESHRE basic semen analysis courses 1995-1999: immediate beneficial effects of standardized training. Hum Reprod 2002; 17: 1299-305.

13 Franken DR, Aneck-Hahn N, Lombaard C, Kruger TF. Semenology training programs: 8 years' experience. Fertil Steril e-pub ahead of print 28 May 2010; PMID 20553675.

14 Pacey AA. Is quality assurance in semen analysis still really necessary? A view from the andrology laboratory. Hum Reprod 2006; 21: 1105-9.

15 Holt WV. Is quality assurance in semen analysis still really necessary? A spermatologist's viewpoint. Hum Reprod 2005; 20: 2983-6.

16 Hargreave TB, Elton RA. Fecundability rates from an infertile male population. $\mathrm{Br} \mathrm{J}$ Urol 1986; 58: 194-7

17 Guzick DS, Overstreet JW, Factor-Litvak P, Brazil CK, Nakajima ST et al. Sperm morphology, motility, and concentration in fertile and infertile men. $N$ Eng/ J Med 2001; 345: 1388-93.

18 Kilani Z, Ismail R, Ghunaim S, Mohamed H, Hughes D et al. Evaluation and treatment of familial globozoospermia in five brothers. Fertil Steril 2004; 82: 1436-9.

19 Tomlinson MJ, Kessopoulou E, Barratt CL. The diagnostic and prognostic value of traditional semen parameters. J Androl 1999; 20: 588-93.

20 Lefièvre L, Bedu-Addo K, Conner SJ, Machado-Oliveira GS, Chen Y et al. Counting sperm does not add up any more: time for a new equation? Reproduction 2007; 133: 675-84.

21 MacLeod J, Gold RZ. The male factor in fertility and infertility. II. Spermatozoon counts in 1000 men of known fertility and in 1000 cases of infertile marriage. $J$ Urol 1951; 66: 436-49.

22 Cooper TG, Noonan E, von Eckardstein S, Auger J, Baker HW et al. World Health Organization reference values for human semen characteristics. Hum Reprod Update 2010; 16: 231-45

23 Ford WC. Comments on the release of the 5th edition of the WHO Laboratory Manual for the Examination and Processing of Human Semen. Asian J Androl 2010; 12: 5963.

24 Baibakov B, Gauthier L, Talbot P, Rankin TL, Dean J. Sperm binding to the zona pellucida is not sufficient to induce acrosome exocytosis. Development 2007; 134 933-43.

25 Liu DY, Liu ML, Baker HW. Enhancement of sperm-zona pellucida (ZP) binding capacity by activation of protein kinase $A$ and $C$ pathways in certain infertile men with defective sperm-ZP binding. Hum Reprod 2009; 24: 20-7.

26 Gupta SK, Bansal P, Ganguly A, Bhandari B, Chakrabarti K. Human zona pellucida glycoproteins: functional relevance during fertilization. J Reprod Immunol 2009; 83 $50-5$

27 Costello S, Michelangeli F, Nash K, Lefievre L, Morris J et al. $\mathrm{Ca}^{2+}$-stores in sperm: their identities and functions. Reproduction 2009; 138: 425-37.

28 Publicover S, Harper CV, Barratt C. Ca2+i signalling in sperm-making the most of what you've got. Nat Cell Biol 2007; 9: 235-242.

29 Krausz C, Bonaccorsi L, Maggio P, Luconi M, Criscuoli L et al. Two functional assays of sperm responsiveness to progesterone and their predictive values in in-vitro fertilization. Hum Reprod 1996; 11:1661-7.

30 Luconi M, Francavilla F, Porazzi I, Macerola B, Forti G et al. Human spermatozoa as a model for studying membrane receptors mediating rapid nongenomic effects of progesterone and estrogens. Steroids 2004; 69: 553-9.

31 Lewis SE. Is sperm evaluation useful in predicting human fertility? Review. Reproduction 2007; 134: 31-40.

32 Dirican EK, Ozgün OD, Akarsu S, Akin KO, Ercan 0 et al. Clinical outcome of magnetic activated cell sorting of non-apoptotic spermatozoa before density gradient centrifugation for assisted reproduction. J Assist Reprod Genet 2008; 25: 375-81.

33 Kheirollahi-Kouhestani M, Razavi S, Tavalaee M, Deemeh MR, Mardani M et al. Selection of sperm based on combined density gradient and Zeta method may improve ICSI outcome. Hum Reprod 2009; 24: 2409-16.

34 Nasr-Esfahani MH, Razavi S, Vahdati AA, Fathi F, Tavalaee M. Evaluation of sperm selection procedure based on hyaluronic acid binding ability on ICSI outcome. J Assist Reprod Genet 2008; 25: 197-203.

35 Parmegiani L, Cognigni GE, Ciampaglia W, Pocognoli P, Marchi F et al. Efficiency of hyaluronic acid (HA) sperm selection. J Assist Reprod Genet 2010; 27: 13-6.

36 Parmegiani L, Cognigni GE, Bernardi S, Troilo E, Ciampaglia W et al. 'Physiologic ICSI': hyaluronic acid (HA) favours selection of spermatozoa without DNA 
Diagnostic tools in male infertility

CLR Barrett et al

58

fragmentation and with normal nucleus, resulting in improvement of embryo quality. Fertil Sterile 2010; 93: 598-604.

37 Bartoov B, Berkovitz A, Eltes F, Kogosovsky A, Yagoda A et al. Pregnancy rates are higher with intracytoplasmic morphologically selection sperm injection than with conventional intracytoplasmic injection. Fertil Sterile 2003; 80(6): 1413-9.

38 Berkovitz A, Eltes F, Yaari S, Katz N, Barr I et al. The morphological normalcy of the sperm nucleus and pregnancy rate of intracytoplasmic injection with morphologically selected sperm. Hum Reprod 2005; 20: 185-90.

39 Hazout A, Dumont-Hassan M, Junca AM, Cohen Bacrie P, Tesarik J. Highmagnification ICSI overcomes paternal effect resistant to conventional ICSI. Reprod Boomed Online 2006; 12: 19-25.

40 Antinori M, Licata E, Bani G, Cerusico F, Versaci C et al. Intracytoplasmic morphologically selected sperm injection: prospective randomized trial. Reprod Boomed Online 2008; 16: 835-41.

41 Cohen J. Cross-overs, sperm redundancy and their close association. Heredity 1973; 31: 408-13.

42 Aitken RJ, Hoppers AJ. Apoptosis and DNA damage in human spermatozoa. Asian J Androl; e-pub ahead of print 30 August 2010; PMID: 20802502.

43 Conner SJ, Lefièvre L, Kirkman-Brown JC, Michelangeli F, Jimenez-Gonzalez C et al. Understanding the physiology of pre-ferilisation events in the human spermatozoa -a necessary prerequisite to developing rational therapy. Soc Reprod Supp/ 2007; 63 : 237-256.

44 Hardy DM, Garbers DL. Species-specific binding of sperm proteins to the extracellular matrix (zona pellucida) of the egg. J Biol Chem 1994; 269: 19000-400.

45 Gao Z, Garbers DL. Species diversity in the structure of zonadhesin, a sperm-specific membrane protein containing multiple cell adhesion molecule-like domains. J Biol Chem 1998; 273: 4315-21.

46 Olson GE, Winfrey VP, Nagdas SK. Acrosome biogenesis in the hamster: ultrastructurally distinct matrix regions are assembled from common precursor polypeptide. Biol Reprod 1998; 58: 361-70.

47 Tardif S, Brady HA, Breazeale KR, Bi M, Thompson LD et al. Zonadhesin D3-polypeptides vary among species but are similar in Equus species capable of interbreeding. Biol Reprod 2010; 82: 413-21.

48 Tardif S, Wilson MD, Wagner R, Hunt P, Gertsenstein M et al. Zonadhesin is essential for species specificity of sperm adhesion to the egg zona pellucida. J Biol Chem 2010; 285: 24863-70.
49 Coppola MA, Klotz KL, Kim KA, Cho HY, Rang J, Shelty J, Howard SS, Flickinger CJ, Herr JC. Sperm Check Fertility, an immunodiagnostic home test that detects normozoospermia and severe oligozoospermia. Hum Reprod 2010; 25: 853-61.

50 Segerink LI, Sprenkels AJ, ter Braak PM, Verges I, van den Berg A. On-chip determination of spermatozoa concentration using electrical impedance measurements. Lab Chip 2010; 10: 1018-24.

51 Bjorndahl L, Kirkman-Brown J, Hart G, Rattle S, Barrett CL. Development of a novel home sperm test. Hum Reprod 2006; 21 : 145-9.

52 Miller D, Tang PZ, Skinner C, Lilford R. Differential RNA fingerprinting as a tool in the analysis of spermatozoal gene expression. Hum Reprod 1994; 9: 864-9.

53 Lalancette C, Miller D, Li Y, Krawetz SA. Paternal contributions: new functional insights for spermatozoal RNA. J Cell Biochem 2008; 104: 1570-9.

54 Baker MA, Reeves G, Hetherington L, Muller J, Bur I et al. Identification of gene products present in the Triton X soluble and insoluble fractions of human spermatozoa lysates using LC-MS/MS analysis. Proteomics Chin App 2007; 1: 524-32.

55 Oxenham SK. Sperm proteomics: thinking outside the collision cell. J Androl 2010; 31: 431-3.

56 Olivia R, de Mate S, Estanyol JM. Sperm cell proteomics. Proteomics 2009; 9: 1004-17.

57 Findlay GD, Swanson WJ. Proteomics enhances evolutionary and functional analysis of reproductive proteins. Bioessays 2010; 32: 26-36.

58 Zhao C, Hus R, Wang FQ, Lin M, Zhou ZM et al. Indentification of several proteins involved in regulation of sperm motility by proteomic analysis. Fertil Sterile 2007; 87: 436-8.

59 Goodsaid FM, Mendrick DL. Translational medicine and the value of biomarker qualification. Sci Trans/ Med 2010; 2: 47ps44.

60 Huser T, Orme CA, Molars CW, Corzett MH, Balhorn R. Raman spectroscopy of DNA packaging in individual human sperm cells distinguishes normal from abnormal cells. J Biophotonics 2009; 2: 322-32.

61 Barratt CL, Aitken RJ, Björndahl L, Carrell DT, de Boer P et al. Sperm DNA: organization, protection and vulnerability: from basic science to clinical applications - a position report. Hum Reprod 2010; 25: 824-38.

62 Glued C, Glued LL. Evidence based diagnostics. BMJ 2005; 330: 724-6.

63 Bossuyt PM, Irwin L, Craig J, Glasziou P. Comparative accuracy: assessing new tests against existing diagnostic pathways. BMJ 2006; 332: 1089-92.

Asian Journal of Andrology 